CASE OF RUPTURE OF THE BLADDER; RECOVERY.

Mr. Chaldecotw records, in a late number of the Provincial Journal, an interesting case of this form of injury. The facts seem too clear to admit of doubt as to the nature of the case. The following is an outline of the history:-

"A healthy and temperate man, of about fifty, having passed two or three hours at a concert, ran across the street to empty his distended bladder, at twelve o'clock at night; and the night being dark, he did not see a newly-erected post, with the top of which the lower part of his abdomen came in violent contact. He states that he fell, and with great diffieulty reached his home, which was about a hundred yards distant.

"Mr. Chaldecott saw him about half an hour after the accident. He was faint, and suffering severe pain over the abdomen, with desire, but no power, to pass his urine. A fullsized catheter was passed easily and completely into the bladder, but no urine escaped. He was placed in bed, and hot fomentations were used to the belly until re-action took place, with which came increase of pain. Twenty leeches were applied, and a gum catheter, but with the same unsatisfactory result as before, not a drop of urine escaping through it.

"Mr. Key being sent for, he arrived about eighteen hours after the accident, by which time the symptoms of peritonitis had increased to an alarming degree. The belly was painful, swollen, and tender; the pulse rapid and feeble; and the countenance anxious. Mr. Key passed a catheter, (none having been used for the previous four hours, and about an ounce of bloody urine came through the instrument. Mr. Key concurred in opinion as to the nature of the injury, and the nearly hopeless prospect for the patient.

"At ten o'clock, he had two scruples of liquor opii sedativus, which, atter a few hours, produced comfortable sleep; and about four hours from the time of $\mathrm{Mr}$. Key's visit, $\mathrm{Mr}$. Chaldecott passed the catheter, and drew off about four ounces of clear urine. From this time, the pain, swelling, and heat in the stomach and abdomen, gradually lessened, and it was evident that the bladder now held, as on each introduction the catheter brought away clear urine.

"From this time until the 13th, (that is, the sixth day from that on which the accident happened,) all went on well, excepting that a smart attack of gout occurred on the $10 \mathrm{th}$, although the patient had never before suffered one; but on the $13 \mathrm{th}$, from a strong desire to become independent of the catheter, he made straining efforts to pass his water, and he had scarcely passed a tablespoonful, when he felt (to use his own expression) something give way, and a burning pain all over his stomach and bowels, as if boiling water had been poured over them, and the same symptoms of faintness and distress occurred as when the accident first happened.

"Mr. Chaldecott saw him within a few minutes of this reopening of the wound of his bladder, for such, no doubt, had been the consequence of his attempts to pass his water. On using the catheter, not more than a teaspoonful came through the tube. He had now again the symptoms of peritonitis, with the addition of incessant sickness. The same plan of treat ment was again adopted-viz., fomentations, leeches, and a full opiate, with calomel.

"About four hours after, on the introduction of the catheter, the bladder was again found to retain the urine; and although the peritonitis had increased to a severe degree, the pain, ten derness, and sickness, gradually subsided; and by a patient submission to the continued use of the catheter for a fortnight, no more interruption to the patient's amendment occurred, excepting that the gont, which, under the use of colchicum, had nearly disappeared, again became severe, no doubt from the fresh absorption of urine which this second accident had permitted to escape into the cavity of the peritonreum. But by this time it was presumed that the wound in the bladder had closed with sufficient firmness to allow the patient to yield to the desire to evacuate his urine without any straining efforts.

"Two months have transpired since the commencement of the case, and he feels no other inconvenience from his accident except a dragging sensation over the abdomen, chiefly on the right side, which is much increased when he attempts to lie upon his left side."

CASE OF ABSCESS OF THE LIVER; OPENED; RECOVERY.

Dr. NorRis records, in the same Journal, a case of abscess of the liver, which well illustrates the advantage of treating such cases by the method recommended by Dr. Greaves-viz., cutting over the cyst of the abscess until the peritonæum is reached; then allowing the matter to form a spontaneous opening. By this means adhesion takes place, and effusion is prevented.

"Thomas P-, aged twenty-nine, car-driver, suffered severely from an affection of the kidneys for six weeks. The symptoms gradually left him, and acute hepatitis immediately followed, which did not yield to the usual remedies. The constitutional symptoms were very severe; hectic fever soon set in, with frequent shiverings; and at the end of six weeks there was a fluctuating tumour in the right hypochondrium, without any external sign of inflammation. The feverish excitement increased, and his sufferings becoming more and more urgent, it appeared certain that the disease had terminated in abscess of the liver. Under these pressing circumstances, Dr. Norris made an incision, an inch and a half ${ }^{-}$in length, through the most prominent part of the swelling down to the peritonxeum; the wound was plugged with a roll of lint to keep it open, and covered with a poultice. At the end of six days, a large quantity of purulent matter, tinged with bile, escaped through the wound. This discharge continued flowing abundantly for a fortnight; the hectic symptoms speedily subsided; the appetite and strength returned. Seven weeks after the operation, he followed his employment, and has enjoyed uninterrupted health seven years, although the wound oozed a little thin fluid for two years before it closed."

\section{MEDDLESOME SURGERY AND ITS EFFECTS.} To the Editor of The Lancex.

Sir,-Will you oblige me by inserting the following case, which, in consequence of a subsequent operation performed by an M.D., has subjected the primary treatment to some dis cussion in this neighbourhood:- On the 13th of June, about six P.M., the Rev. J.J., while walking beside some mowers, in a hay-field, received a punctured wound from a scythe on the outer aspect of the leg, about an inch below the calf. When I reached the spot, I found that profuse hæmorrhage had taken place, which was arrested by the compression of a workman's finger pressed into the wound. Being then in attendance on a case of midwifery, $I$ could do nothing more than bind up the wound, order the patient to be carefully conveyed home, and the leg to be kept wetted with cold lotion during the night.

I was not able to visit him until nine o'clock the following morning. I found the leg rather swollen and painful; no hæemorrhage had occurred, not even sufficient to tinge the bandages with the colour of blood; the wound slightly pulsated. As his general health was very infirm, his temperament nervous and irritable, and he was a constant sufferer from irregular gouty action, which, with other diseases, had reduced him to a state of emaciation, rendering him a very unfit subject for an operation, I determined upon healing the wound by adhesion.

From this period, everything progressed favourably; not the slightest hæmorrhage occurred; and in a fortnight, although contrary to my advice, he resumed his public duties.

I have subsequently learned that an operation was undertaken by an M.D. in the neighbourhood, who was in occasional attendance on some members of the family. A fresh wound was made on the opposite side of the leg. Nearly three hours were occupied in securing the artery, (posterior tibial;) while a retired surgeon, who was present, triumphantly produced two or three pieces of coagula, which were declared throughout the country to be

"More venomous than the serpents of the Nile."

The consequences were such as might have been anticipated by those acquainted with the debilitated, shattered state of the unfortunate sufferer's nervous system. He gradually sank, and died in a few weeks afterwards.

Now, Sir, will you have the goodness to inform me what pathologist ever ascribed tremendous irritation to the presence of a clot of blood, or what M.D., in the plenitude of surgical skill, would employ such a hazardous operation, in its ultimate effects, for the removal of a mere thrombus Speaking of coagula, the highest surgical authority in this kingdom-viz., Professor Cooper, of University College, London - says, "I have never seen any case in which there was any real occasion to make an opening for the discharge of the blood." Thomas EdTards, M.R.C.S. 